\title{
Non-covalent functionalization of single wall carbon nanotubes and graphene by a conjugated polymer
}

Jiwuer Jilili, Ayjamal Abdurahman, Oğuz Gülseren, and Udo Schwingenschlögl

Citation: Appl. Phys. Lett. 105, 013103 (2014);

View online: https://doi.org/10.1063/1.4886968

View Table of Contents: http://aip.scitation.org/toc/apl/105/1

Published by the American Institute of Physics

\section{Articles you may be interested in}

A consistent and accurate ab initio parametrization of density functional dispersion correction (DFT-D) for the 94 elements $\mathrm{H}-\mathrm{Pu}$

The Journal of Chemical Physics 132, 154104 (2010); 10.1063/1.3382344

Noncovalent functionalization of carbon nanotubes by aromatic organic molecules

Applied Physics Letters 82, 3746 (2003); 10.1063/1.1577381

Carbon nanotube $p-n$ junction diodes

Applied Physics Letters 85, 145 (2004); 10.1063/1.1769595

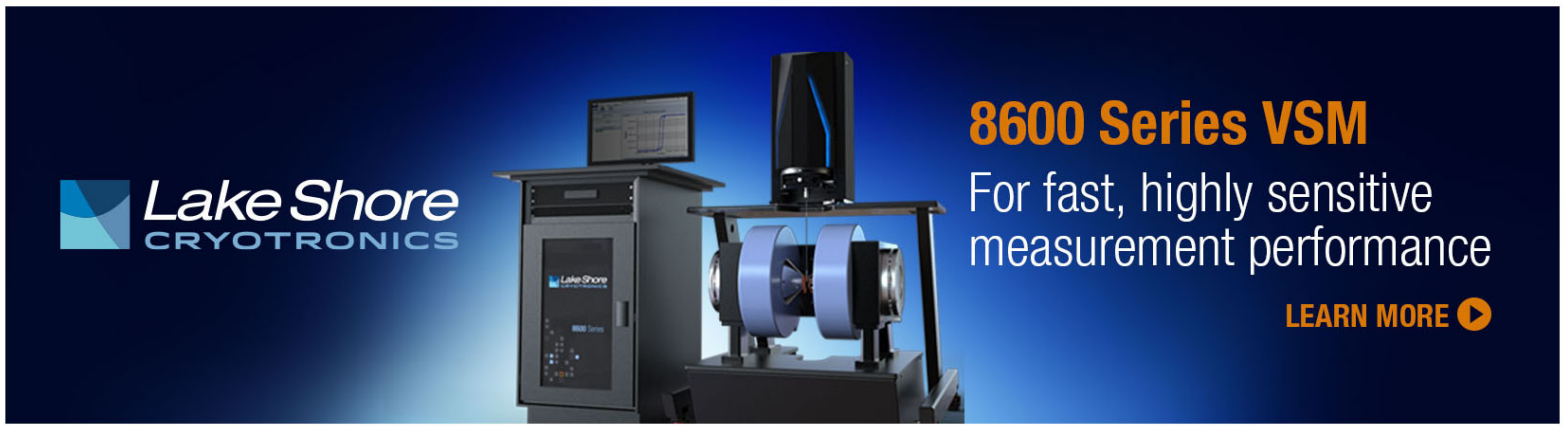




\title{
Non-covalent functionalization of single wall carbon nanotubes and graphene by a conjugated polymer
}

\author{
Jiwuer Jilili, ${ }^{1}$ Ayjamal Abdurahman, ${ }^{2}$ Oğuz Gülseren, ${ }^{2}$ and Udo Schwingenschlögl ${ }^{1, a)}$ \\ ${ }^{1}$ KAUST, PSE Division, Thuwal 23955-6900, Kingdom of Saudi Arabia \\ ${ }^{2}$ Department of Physics, Bilkent University, 06800 Ankara, Turkey
}

(Received 2 March 2014; accepted 22 June 2014; published online 7 July 2014)

\begin{abstract}
We report first-principles calculations on the binding of poly[(9,9-bis-(6-bromohexylfluorene-2,7diyl)-co-(benzene-1,4-diyl)] to a $(8,0)$ single wall carbon nanotube (SWCNT) and to graphene. Considering different relative orientations of the subsystems, we find for the generalized gradient approximation a non-binding state, whereas the local density approximation predicts reasonable binding energies. The results coincide after inclusion of van der Waals corrections, which demonstrates a weak interaction between the polymer and SWCNT/graphene, mostly of van der Waals type. Accordingly, the density of states shows essentially no hybridization. The physisorption mechanism explains recent experimental observations and suggests that the conjugated polymer can be used for non-covalent functionalization. (C) 2014 AIP Publishing LLC. [http://dx.doi.org/10.1063/1.4886968]
\end{abstract}

Carbon nanotubes (CNTs) have potential for reinforcing polymer composites due to unique properties such as a high aspect ratio, low density, and high mechanical and thermal stability. ${ }^{1,2}$ Surface modification of CNTs by covalent or noncovalent functionalization is a powerful and viable strategy for developing high performance composite materials, chemosensors, nanoelectronics, photovoltaic devices, ${ }^{3,4}$ and a range of biomedical applications. ${ }^{5,6}$ Graphene, ${ }^{7}$ a two-dimensional honeycomb sheet of $s p^{2}$-bonded $\mathrm{C}$ atoms, can be regarded as the basic building unit of CNTs where the detailed reactivity is still not well understood. Functionalization of graphene is also promising for applications, particularly in the area of polymer nanocomposites. 8,9

It is widely accepted that chemical modification of CNTs with functional monomers and polymers or physical wrapping over the CNT are appropriate methods for fabricating hybrid materials with tailored properties and functionalities. $^{10-13}$ In general, polymers can interact with CNTs or graphene via strong covalent ${ }^{14}$ or electrostatic interactions, ${ }^{15}$ $\pi$-stacking, ${ }^{16,17}$ hydrogen bonding, ${ }^{18}$ or weak van der Waals (vdW) interactions. ${ }^{19,20}$ Non-covalent functionalization with conjugated polymers is particularly attractive, because chemical groups are attached without disrupting the bonding network of the CNT, whereas covalent functionalization introduces atomic defects and internal stress, which can deteriorate the mechanical properties. ${ }^{21}$ Unique electrical and optical properties have been demonstrated for CNTs interacting with conjugated polymers. $^{22,23}$

It is desirable to optimize the non-covalent intermolecular interactions in view of achieving a strong interfacial binding in the composite. Previous theoretical studies have addressed this issue using molecular dynamic simulations as well as density functional theory (DFT). ${ }^{22-29}$ For instance, the possibility to improve the polymer-CNT alignment by suitable tuning of the temperature, polymer density, and chain length has been demonstrated in Ref. 25. In Ref. 26,

\footnotetext{
a) Telephone: +966(0)544700080. Electronic mail: udo.schwingenschlog1@ kaust.edu.sa.
}

the covalent and non-covalent surface functionalization of metallic and semiconducting CNTs by Fe-porphyrin has been studied. In Ref. 27, the authors have demonstrated that CNTs coated with alginic acid are soluble in water and interact via vdW forces. Moreover, the authors of Ref. 28 have investigated the solubility of CNTs wrapped with chitosan to identify the relevant interactions. In general, the orientation of polymers on carbon nanostructures also depends on the flexibility of the polymer backbone. ${ }^{30}$ The interaction of poly[(9,9-bis-(6-bromohexylfluorene-2,7-diyl)-co-(benzene1,4-diyl)] with single wall CNTs (SWCNTs) has not yet been studied, though experimentally this polymer was found to be suitable for non-covalent functionalization ${ }^{31}$ (where the nature of the interaction remained dubious and calls for clarification). We therefore investigate in the following in detail the interaction of this polymer with a SWCNT, applying pseudopotential plane wave density functional theory. In addition, we address the interaction with graphene as prototypical two-dimensional material.

Density functional theory is a well established theoretical method providing an accurate description of covalent and ionic chemical bonds. On the other hand, it is more involved to reproduce nonlocal dispersive forces, in particular vdW forces which, however, are important in weakly bonded systems. Although the local density approximation (LDA) tends to overestimate binding energies between molecules and the generalized gradient approximation (GGA) often underestimates them, we will demonstrate that density functional theory for the present system leads to a favorable balance between accuracy and computational efficiency. Usage of more sophisticated methods, such as high level wave function based methods, in general, would be desirable, but due to the huge computational costs is unrealistic for systems as large as those considered here.

We have performed calculations using both the LDA in the Perdew-Zunger parametrization ${ }^{32}$ and the GGA in the Perdew-Burke-Ernzerhof parametrization. ${ }^{33} \mathrm{We}$ will argue that the LDA yields a correct binding energy trend for the different orientations of the polymer with respect to the 
SWCNT/graphene. The pure GGA results in nonbinding states, whereas binding energies obtained under inclusion of the DFT-D vdW correction ${ }^{34-36}$ reproduce the LDA trend for all considered cases. All calculations are based on the projector augmented plane wave method ${ }^{37,38}$ of the Vienna Ab-initio Simulation Package.

The polymer is represented by one monomer, as shown in Fig. 1, which is justified by the fact that the electronic gaps of the monomer and the dimer are found to deviate by only $0.17 \mathrm{eV}$. Our supercells consist of a semiconducting $(8,0)$ SWCNT and the monomer, see Figs. 2(a)-2(d), or a graphene sheet $(10 \times 10)$ and the monomer, see Figs. $3(a)-3($ c). We choose a zigzag $(8,0)$ SWCNT because of its semiconducting property and intermediate diameter, which combines a small curvature (resembling graphene) with reduced computational costs. A similar approach for evaluating the interaction between semiconducting conjugated polymers (para-phenylene and para-borazylene) and SWCNTs has been reported in Ref. 39. We consider various orientations of the monomer with respect to the SWCNT/graphene and optimize for each of them the atomic positions by the conjugate gradient method with an energy threshold of $10^{-5} \mathrm{eV}$. We also optimize the monomer, SWCNT, and graphene individually to evaluate the structural modifications due to the interaction in the joined systems. Careful convergence tests have been performed for both the energy cutoff and the k-point sampling. For the structure relaxation we employ $1 \times 1 \times 1$ and $4 \times 4 \times 1$ Monkhorst-Pack grids ${ }^{40}$ for the monomer-SWCNT and monomer-graphene systems, respectively, whereas fine $1 \times 1 \times 11$ and $13 \times 13 \times 1$ grids are used for calculating energies and densities of states. A plane wave energy cutoff of $450 \mathrm{eV}$ is used and periodic boundary conditions are applied, where each supercell contains a $10 \AA$ vacuum slab along the c-axis. The binding energy between the two subsystems is calculated as

$$
E_{b}=E_{\text {monomer-SWCNT/graphene }}-E_{S W C N T / \text { graphene }}-E_{\text {monomer }},
$$

where $E_{\text {monomer-SWCNT/graphene }}, E_{S W C N T / \text { graphene }}$, and $E_{\text {monomer }}$ denote the total energies of the joint system and of the components.

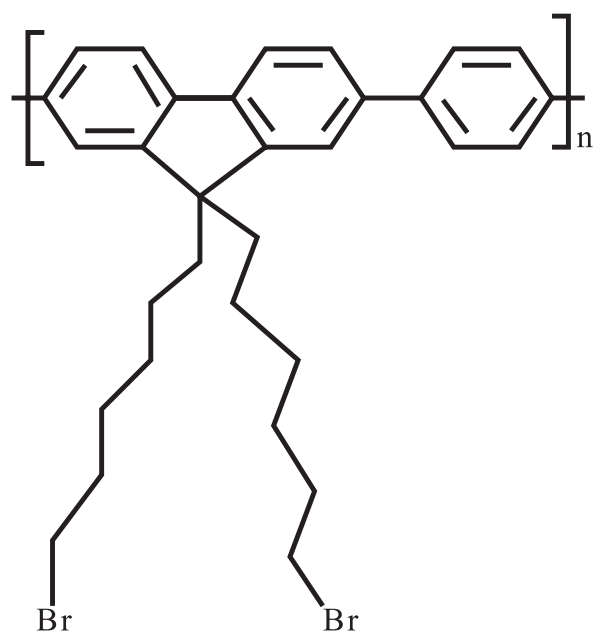

FIG. 1. Structure of conjugated poly[(9,9-bis-(6-bromohexylfluorene-2,7diyl)-co-(benzene-1,4-diyl)].

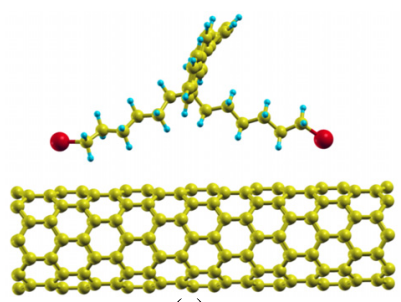

(a)

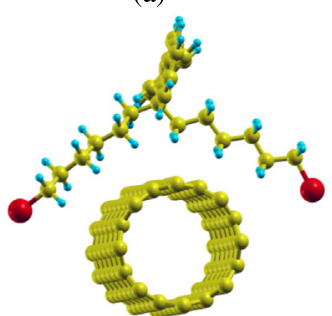

(c)

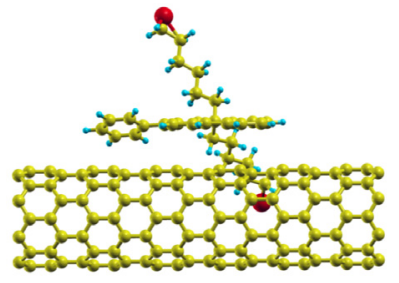

(b)

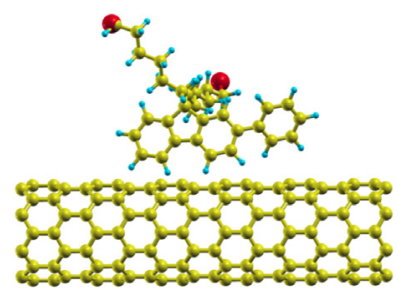

(d)
FIG. 2. (a)-(d) Configurations studied for the monomer-SWCNT system.

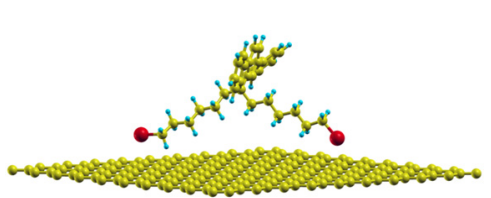

(a)

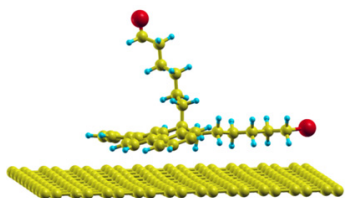

(b)

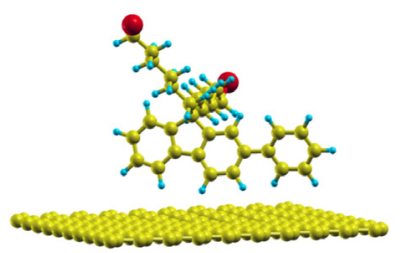

(c)

FIG. 3. (a)-(c) Configurations studied for the monomer-graphene system.

Various approaches have been suggested to treat dispersion interactions within density functional theory. ${ }^{36,41-44}$ Those based on dispersion correction functionals, such as the method of Dion and coworkers, ${ }^{41}$ are typically connected with high computational costs for larger systems. ${ }^{45}$ Grimme $^{34,35}$ has developed a method that adds an empirical correction to the standard density functional. Thus, the total energy is given by

$$
E_{D F T-D}=E+E_{\text {dispersion }},
$$

where $E$ is the self-consistent Kohn-Sham energy as obtained from the chosen density functional and $E_{\text {dispersion }}$ is the empirical correction given by

$$
E_{\text {dispersion }}=-s_{6} \sum_{i=1}^{N-1} \sum_{j=i+1}^{N} \frac{C_{6}^{i j}}{R_{i j}^{6}} f_{\text {damping }}\left(R_{i j}\right) .
$$

Here, $s_{6}$ is a global scaling factor that only depends on the density functional, $C_{6}^{i j}$ denotes the dispersion coefficient for the pair $i j$, and $R_{i j}$ is the internuclear distance. In addition, a damping function $f_{\text {damping }}$ is introduced to avoid singularities for small $R_{i j}$. The Becke-Johnson damping has been found to give the best results for nonbonded distances ${ }^{46}$ and therefore is applied in our study in the latest DFT-D3 version of the Grimme method. ${ }^{36}$ Although this method excludes collective 
quantum mechanical effects, it is one of the most widely applied and well tested schemes ${ }^{43,46}$ and has been applied successfully to small molecular adducts, $\pi$-stacking systems, and large complexes in biological systems. ${ }^{47}$ Further examples for accurate results on related molecules can be found in Refs. 48-50. Recently, Rahman and colleagues ${ }^{51}$ have investigated nucleobases on graphene using several variants of vdW corrections. The authors have concluded that the Grimme method is an excellent choice for modeling the interaction between molecules and graphene or CNTs.

The structural modifications due to the interaction between the monomer and SWCNT are very small for all configurations shown in Fig. 2, which represent prototypical orientations between the two subsystems. Calculated binding energies at the LDA, GGA, and GGA + vdW levels, minimal distances between monomer and SWCNT, and results of Bader charge transfer analyses are listed in Table I. The LDA and GGA binding energies are found to be fundamentally different for all cases. Pure GGA does not yield any binding, whereas under inclusion of the vdW correction the LDA trend is recovered. This result is consistent with previous observations that in $\mathrm{vdW}$ systems the LDA provides a reasonable description of dispersive interactions. ${ }^{52-55}$

For the configuration of Fig. 2(a), the LDA binding energy is $-0.30 \mathrm{eV}$ and the shortest distance between the non-hydrogen atoms of the monomer and the SWCNT is $3.26 \AA$. The monomer chain thus interacts only weakly with the SWCNT, which is in agreement with earlier theoretical predictions of Cohen and coworkers ${ }^{56}$ and results for atomic $\mathrm{Br}$ adsorbed on a SWCNT. ${ }^{57}$ When the monomer backbone is oriented parallel to the SWCNT, see Fig. 2(b), the interaction is slightly stronger with a binding energy of $-0.44 \mathrm{eV}$. It is mediated by $\pi$-orbitals of the monomer aromatic ring and the SWCNT. This situation is similar to other $\pi$-stacking systems, such as bilayer graphene, ${ }^{52,58}$ and the $\pi$-interaction between SWCNTs and conjugated organic polymers ${ }^{39}$ and molecules. ${ }^{17,54}$ When the monomer approaches with the chain side and wraps around the SWCNT, see Fig. 2(c), the binding energy is less negative $(-0.39 \mathrm{eV})$ with a minimal $\mathrm{C}-\mathrm{C}$ distance of $3.34 \AA$. The dominance of $\mathrm{vdW}$ forces in all the configurations is reflected by equilibrium distances within the typical vdW range. The fact that the LDA and GGA $+\mathrm{vdW}$ methods predict similar trends for the binding energies of the different configurations indicates that our results are accurate and yield solid conclusions.

We next analyze the electronic density of states in Fig. 4. All configurations reproduce the main features of the pristine SWCNT, indicating that the electronic properties of

TABLE I. Binding energies $E_{b}$ (in eV) for the orientations shown in Fig. 2, minimum distance $d_{\text {min }}$ (in $\AA$ ) between monomer and SWCNT (excluding $\mathrm{H}$ atoms), and charge transfer $\Delta Q$ (in electrons).

\begin{tabular}{llllcc}
\hline \hline & \multicolumn{3}{c}{ LDA } & GGA & GGA + vdW \\
\cline { 2 - 4 } Configuration & $E_{b}$ & $d_{\text {min }}$ & $\Delta Q$ & $E_{b}$ & $E_{b}$ \\
\hline Fig. 2(a) & -0.30 & 3.26 & 0.01 & 0.05 & -0.46 \\
Fig. 2(b) & -0.44 & 3.13 & 0.03 & 0.27 & -0.51 \\
Fig. 2(c) & -0.39 & 3.34 & -0.02 & 0.08 & -0.62 \\
Fig. 2(d) & -0.13 & 3.32 & -0.01 & 0.10 & -0.23 \\
\hline \hline
\end{tabular}
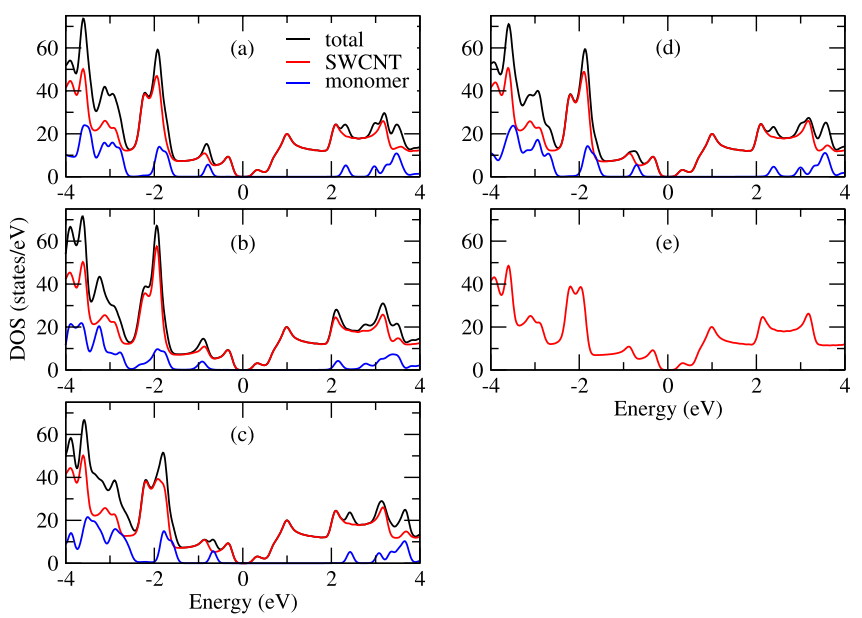

FIG. 4. (a)-(d) Densities of states for the monomer-SWCNT system calculated at the LDA level for the configurations of Fig. 2. (e) Corresponding results for the pristine SWCNT.

the SWCNT are well maintained in the combined systems. We find no indication of hybridization between atoms of the SWCNT and monomer, because the corresponding densities of states show no common peaks. The charge transfer from the monomer to the SWCNT in the configurations of Figs. 2(a) and 2(b) turns out to be 0.01 and 0.03 electrons, whereas for the configurations of Figs. 2(c) and 2(d) it is 0.02 and 0.01 electrons in the opposite direction. This observation supports the conclusion that the monomer and SWCNT interact weakly and indicates that the conjugated polymer has potential as wrapping material for non-covalent functionalization of SWCNTs. According to Table I, the binding energy per atom involved in the interaction is estimated to be of the same order of magnitude but still larger than the thermal energy at room temperature. Our results thus are comparable to values reported for other polymers that can be used for non-covalent functionalization. ${ }^{59,60}$

Turning to the monomer-graphene systems, we next analyze the configurations shown in Fig. 3. In panel (a), the monomer chains point towards the graphene, whereas in (b) and (c) the monomer backbone is aligned parallel and perpendicular to the sheet, respectively. In contrast to the monomer-SWCNT systems, we observe structural distortions due to the interaction between the two components. When the monomer backbone comes close to the graphene, see Figs. 3(b) and 3(c), the sheet bends locally, reflected by atomic shifts of up to $0.03 \AA$ with respect to the original positions. Similarly to the monomer-SWCNT systems, we find for all configurations that the pure GGA yields non-binding states, whereas the LDA and GGA + vdW methods result in the same trends for the binding energies, see Table II. We obtain $-0.34 \mathrm{eV}$ for the configuration of Fig. 3(a), as the binding between $\mathrm{Br}$ and the $\mathrm{C}$ atoms in the alkene is strong enough to inhibit bond formation to the graphene sheet. For the configurations of Figs. 3(b) and 3(c), we obtain binding energies of $-0.76 \mathrm{eV}$ and $-0.35 \mathrm{eV}$, respectively. The authors of Ref. 61 have demonstrated strong $\pi-\pi$ interaction between the backbones of conjugated polymers and graphene, which agrees with our finding of an enhanced binding energy when the monomer backbone is aligned parallel to the graphene sheet. The situation is similar to the interaction 
TABLE II. Binding energies $E_{b}$ (in eV) for the orientations shown in Fig. 3, minimum distance $d_{\text {min }}$ (in $\AA$ ) between monomer and graphene (excluding $\mathrm{H}$ atoms), and charge transfer $\Delta Q$ (in electrons).

\begin{tabular}{|c|c|c|c|c|c|}
\hline \multirow[b]{2}{*}{ Configuration } & \multicolumn{3}{|c|}{ LDA } & \multirow{2}{*}{$\begin{array}{c}\text { GGA } \\
E_{b}\end{array}$} & \multirow{2}{*}{$\begin{array}{c}\mathrm{GGA}+\mathrm{vdW} \\
E_{b}\end{array}$} \\
\hline & $E_{b}$ & $d_{\min }$ & $\Delta Q$ & & \\
\hline Fig. 3(a) & -0.34 & 3.22 & 0.02 & 0.01 & -0.64 \\
\hline Fig. 3(b) & -0.76 & 3.19 & 0.01 & 0.37 & -0.88 \\
\hline Fig. 3(c) & -0.35 & 3.29 & -0.02 & 0.00 & -0.68 \\
\hline
\end{tabular}
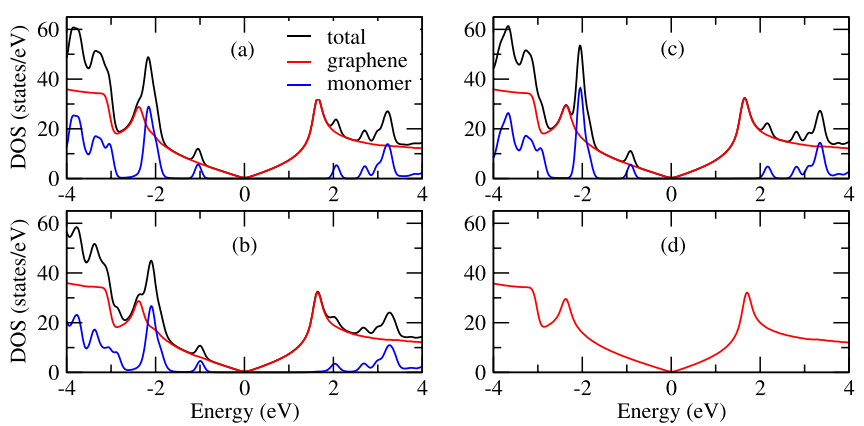

FIG. 5. (a)-(c) Densities of states for the monomer-graphene system calculated at the LDA level for the configurations of Fig. 3. (d) Corresponding results for pristine graphene.

of graphene with polymers and molecules. ${ }^{39,54,62}$ Bader analyses show for no configuration a significant charge transfer and the densities of states in Fig. 5 demonstrate that there is little interaction between orbitals of the monomer and the graphene, as there is essentially no hybridization visible.

We have performed first-principles calculations using the pseudopotential plane wave method to study the interaction of a conjugated polymer with a SWCNT as well as with graphene. Various relative orientations of the two subsystems have been considered. Our results show that the GGA is not appropriate for describing the systems under study, whereas the LDA and the vdW corrected GGA give similar and conclusive results. The densities of states obtained for the composites and their subsystems reveal that the electronic structure of the SWCNT/graphene is well maintained, in particular around the Fermi energy, when the polymer is attached. This finding confirms the experimental observation that the polymer is suitable for non-covalent functionalization. In addition, its hydrophilic nature ${ }^{31}$ indicates that the functionalization in the present case is not driven by interaction with the solvent. According to Bader analyses, the charge transfer between the subsystems is negligible. The polymer-SWCNT/graphene interaction thus is of weak vdW type with only minor effects on the physical and electronic properties of the SWCNT/graphene, which is important for an effective non-covalent functionalization. Finally, a physisorption mechanism is confirmed by the obtained binding energies and densities of states. We expect that the derived results can serve as reference for work on related polymers.

A.A. thanks for the support of TUBITAK (Grant No. TBAG-107T892), the European Union Framework 7 Project Unam-Regpot (Grant No. 203953), and S. Ehrlich for technical support with the DFT-D code and for helpful discussions.
Computational resources have been provided by KAUST HPC and IT. Research reported in this publication was supported by the King Abdullah University of Science and Technology (KAUST).

${ }^{1}$ M. F. Yu, B. S. Files, S. Arepalli, and R. S. Ruoff, Phys. Rev. Lett. 84, 5552-5555 (2000).

${ }^{2}$ M. Moniruzzaman and K. I. Winey, Macromolecules 39, 5194-5205 (2006).

${ }^{3}$ T. Schuettfort, A. Nish, and R. J. Nicholas, Nano Lett. 9, 3871-3876 (2009).

${ }^{4}$ R. C. Tenent, T. M. Barnes, J. D. Bergeson, A. J. Ferguson, B. To, L. M. Gedvilas, M. J. Heben, and J. L. Blackburn, Adv. Mater. 21, 3210-3216 (2009).

${ }^{5}$ D. Tasis, N. Tagmatarchis, A. Bianco, and M. Prato, Chem. Rev. 106, 1105-1136 (2006).

${ }^{6}$ E. Mehdipoor, M. Adeli, M. Bavadi, P. Sasanpour, and B. Rashidian, J. Mater. Chem. 21, 15456-15463 (2011).

${ }^{7}$ A. K. Geim, Science 324, 1530-1534 (2009).

${ }^{8}$ H. Kim, A. A. Abdala, and C. W. Macosko, Macromolecules 43, 6515-6530 (2010).

${ }^{9}$ J. R. Potts, D. R. Dreyer, C. W. Bielawski, and R. S. Ruoff, Polymer 52, 5-25 (2011).

${ }^{10}$ Y. Lin, M. J. Meziani, and Y.-P. Sun, J. Mater. Chem. 17, 1143-1148 (2007).

${ }^{11}$ H. Ago, K. Petritsch, M. S. P. Shaffer, A. H. Windle, and R. H. Friend, Adv. Mater. 11, 1281-1285 (1999).

${ }^{12}$ Y. Zeng, P. Liu, J. Du, L. Zhao, P. M. Ajayan, and H.-M. Cheng, Carbon 48, 3551-3558 (2010).

${ }^{13}$ J. Zou, L. Liu, H. Chen, S. I. Khondaker, R. D. McCullough, Q. Hou, and L. Zhai, Adv. Mater. 20, 2055-2060 (2008).

${ }^{14}$ H. Kitano, K. Tachimoto, M. G. Ide, and N. Tsubaki, Macromol. Chem. Phys. 207, 812-819 (2006).

${ }^{15}$ J. Bae, S. H. Cha, and J. Park, Macromol. Res. 21, 826-831 (2013).

${ }^{16}$ L. Q. Xu, L. Wang, B. Zhang, C. H. Lim, Y. Chen, K. G. Neoh, E. T. Kang, and G. D. Fu, Polymer 52, 2376-2383 (2011).

${ }^{17}$ C. Roquelet, B. Langlois, F. Vialla, D. Garrot, J. S. Lauret, and C. Voisin, Chem. Phys. 413, 45-54 (2013).

${ }^{18}$ J. Liang, Y. Huang, L. Zhang, Y. Wang, Y. Ma, T. Guo, and Y. Chen, Adv. Funct. Mater. 19, 2297-2302 (2009).

${ }^{19}$ E. K. Hobbie, T. Ihle, J. M. Harris, and M. R. Semler, Phys. Rev. B 85, 245439 (2012).

${ }^{20}$ M. Hassan, M. Walter, and M. Moseler, Phys. Chem. Chem. Phys. 16, 33-37 (2014).

${ }^{21}$ M. T. Byrne and Y. K. Gunko, Adv. Mater. 22, 1672-1688 (2010).

${ }^{22}$ Y. K. Kang, O.-S. Lee, P. Deria, S. H. Kim, T.-H. Park, D. A. Bonnell, J. G. Saven, and M. J. Therien, Nano Lett. 9, 1414-1418 (2009).

${ }^{23}$ M. G. Ahangari, A. Fereidoon, and M. D. Ganji, J. Mol. Model. 19, 3127-3134 (2013).

${ }^{24}$ M. Yang, V. Koutsos, and M. Zaiser, J. Phys. Chem. B 109, 10009-10014 (2005).

${ }^{25}$ C. Caddeo, C. Melis, L. Colombo, and A. Mattoni, J. Phys. Chem. C 114, 21109-21113 (2010).

${ }^{26}$ I. R. Tagle and W. Orellana, Phys. Rev. B 82, 115406 (2010).

${ }^{27}$ Y. Z. Liu, C. Chipot, X. G. Shao, and W. S. Cai, J. Phys. Chem. B 114, 5783-5789 (2010).

${ }^{28}$ T. Rungrotmongkol, U. Arsawang, C. Iamsamai, A. Vongachariya, S. T. Dubas, U. Ruktanonchai, A. Soottitantawat, and S. Hannongbua, Chem. Phys. Lett. 507, 134-137 (2011).

${ }^{29}$ L. Y. Xu and X. N. Yang, J. Colloid Interface Sci. 418, 66-73 (2014).

${ }^{30}$ I. Gurevitch and S. Srebnik, Chem. Phys. Lett. 444, 96-100 (2007).

${ }^{31}$ B. Baykal, V. Ibrahimova, G. Er, E. Bengu, and D. Tuncel, Chem. Commun. 46, 6762-6764 (2010).

${ }^{32}$ J. P. Perdew and A. Zunger, Phys. Rev. B 23, 5048-5079 (1981).

${ }^{33}$ J. P. Perdew, K. Burke, and M. Ernzerhof, Phys. Rev. Lett. 77, 3865-3868 (1996).

${ }^{34}$ S. Grimme, J. Comput. Chem. 25, 1463-1476 (2004).

${ }^{35}$ S. Grimme, J. Comput. Chem. 27, 1787-1799 (2006).

${ }^{36}$ S. Grimme, J. Antony, S. Ehrlich, and H. Krieg, J. Chem. Phys. 132, 154104 (2010).

${ }^{37}$ P. E. Blöchl, Phys. Rev. B 50, 17953-17979 (1994).

${ }^{38}$ G. Kresse and D. Joubert, Phys. Rev. B 59, 1758-1775 (1999).

${ }^{39}$ M. Simeoni, C. De Luca, S. Picozzi, S. Santucci, and B. Delley, J. Chem. Phys. 122, 214710 (2005). 
${ }^{40}$ H. J. Monkhorst and J. D. Pack, Phys. Rev. B 13, 5188-5192 (1976).

${ }^{41}$ M. Dion, H. Rydberg, E. Schröder, D. C. Langreth, and B. I. Lundqvist, Phys. Rev. Lett. 92, 246401 (2004).

${ }^{42}$ A. D. Becke and E. R. Johnson, J. Chem. Phys. 123, 154101 (2005).

${ }^{43}$ S. Grimme, J. Antony, T. Schwabe, and C. Muck-Lichtenfeld, Org. Biomol. Chem. 5, 741-758 (2007).

${ }^{44}$ A. Tkatchenko and M. Scheffler, Phys. Rev. Lett. 102, 073005 (2009).

${ }^{45}$ J. Gräfenstein and D. Cremer, J. Chem. Phys. 130, 124105 (2009).

${ }^{46}$ S. Grimme, S. Ehrlich, and L. Goerigk, J. Comput. Chem. 32, 1456-1465 (2011).

${ }^{47}$ B. Civalleri, C. M. Zicovich-Wilson, L. Valenzanoa, and P. Ugliengo, CrystEngComm 10, 405-410 (2008).

${ }^{48}$ M. Parac, M. Etinski, M. Peric, and S. Grimme, J. Chem. Theory Comput. 1, 1110-1118 (2005).

${ }^{49}$ M. Piacenza and S. Grimme, J. Am. Chem. Soc. 127, 14841-14848 (2005).

${ }^{50}$ J. Antony and S. Grimme, Phys. Chem. Chem. Phys. 8, 5287-5293 (2006).

${ }^{51}$ D. Le, A. Kara, E. Schröder, P. Hyldgaard, and T. S. Rahman, J. Phys.: Condens. Matter 24, 424210 (2012).

${ }^{52}$ L. A. Girifalco and M. Hodak, Phys. Rev. B 65, 125404 (2002).
${ }^{53}$ F. Tournus and J.-C. Charlier, Phys. Rev. B 71, 165421 (2005).

${ }^{54}$ F. Tournus, S. Latil, M. I. Heggie, and J.-C. Charlier, Phys. Rev. B 72, 075431 (2005).

${ }^{55}$ L. M. Woods, Ş. C. Bădescu, and T. L. Reinecke, Phys. Rev. B 75, 155415 (2007).

${ }^{56}$ S.-H. Jhi, S. G. Louie, and M. L. Cohen, Solid State Commun. 123, 495-499 (2002).

${ }^{57}$ N. Park, Y. Miyamoto, K. Lee, W. Choi, J. Ihm, J. Yu, and S. Han, Chem. Phys. Lett. 403, 135-139 (2005).

${ }^{58}$ F. Tran, R. Laskowski, P. Blaha, and K. Schwarz, Phys. Rev. B 75, 115131 (2007).

${ }^{59}$ F. F. Contreras-Torres and E. Martínez-Lorán, Wiley Interdiscip. Rev.: Comput. Mol. Sci. 1, 902-919 (2011).

${ }^{60}$ S. I. Pascu, N. Kuganathan, L. H. Tong, R. M. J. Jacobs, P. J. Barnard, B. T. Chu, Y. Huh, G. Tobias, C. G. Salzmann, J. K. M. Sanders, M. L. H. Green, and J. C. Green, J. Mater. Chem. 18, 2781-2788 (2008).

${ }^{61}$ H. Bai, Y. Xu, L. Zhao, C. Li, and G. Shi, Chem. Commun. 13, 1667-1669 (2009).

${ }^{62}$ J. Bjork, F. Hanke, C. A. Palma, P. Samori, M. Cecchini, and M. Persson, J. Phys. Chem. Lett. 1, 3407-3412 (2010). 